\title{
Optimal Feedback Schemes Over Unknown Channels
}

\author{
Aslan Tchamkerten and Emre I. Telatar ${ }^{1}$ \\ Information Theory Laboratory (LTHI) \\ School of Computer and Communication Sciences (I\&C) \\ Swiss Federal Institute of Technology of Lausanne (EPFL) \\ CH-1015 Lausanne, Switzerland \\ \{aslan.tchamkerten, emre.telatar\}@epfl.ch
}

\begin{abstract}
Communication over unknown discrete memoryless channels with instantaneous and perfect feedback is considered. For a given set of channels we define a notion of optimal coding schemes in terms of achievable rate and error exponent, and prove the existence of such coding schemes for two families of channels.
\end{abstract}

It is well known that the capacity of a discrete memoryless channel (DMC) cannot be increased by means of perfect and instantaneous feedback. However, when perfect feedback is available a significant gain in terms of the error exponent is possible. In 1976, Burnashev [1] computed the maximum achievable error exponent for DMC's with perfect and instantaneous feedback using variable length codes to be

$$
E_{B}\left(R, Q_{Y \mid X}\right)=\left(\max _{x, x^{\prime}} D\left(Q_{Y \mid x} \| Q_{Y \mid x^{\prime}}\right)\right)\left(1-\frac{R}{C\left(Q_{Y \mid X}\right)}\right),
$$

where the maximization is over all pairs of input symbols, $R$ is the communication rate and $C\left(Q_{Y \mid X}\right)$ the capacity of the channel $Q_{Y \mid X}{ }^{2}$. It is important to note that both the rate and the error exponent are with respect to the expected codeword length.

We study the situation where communication is carried over a DMC with time invariant transition probability matrix $Q_{Y \mid X}$ that is unknown to both the transmitter and the receiver. However, we make the assumption that transmitter and receiver have the knowledge that $Q_{Y \mid X}$ belongs to some subset $\mathcal{Q}$ of DMC's.

For sake of clarity, definitions 1,2 and 3 review the notions of coding scheme, rate and error exponent related to feedback communication. Definition 4 introduces a notion of optimal sequences of coding schemes and is followed by our main result.

Definition 1 (Coding Scheme). For any message set $\mathcal{M}$ of size $M \geq 2$, an encoder is a sequence of functions

$$
\Phi^{M}=\left\{X_{n}: \mathcal{M} \times \mathcal{Y}^{n-1} \longrightarrow \mathcal{X}\right\}_{n \geq 1} .
$$

For a message $m$, the symbol $x_{n}$ to be sent at time $n$ is given by $X_{n}\left(m, y_{1}^{n-1}\right)$ where $y_{1}^{n-1}=y_{1}, y_{2}, \ldots, y_{n-1}$ denotes the received symbols up to time $n-1$.

A decoder $\left(\Psi^{M}, U(M)\right)$ consists of a set of functions

$$
\Psi^{M}=\left\{\psi_{n}^{M}: \mathcal{Y}^{n} \longrightarrow \mathcal{M}\right\}_{n \geq 1},
$$

${ }^{1}$ The work presented in this paper was supported (in part) by the National Competence Center in Research on Mobile Information and Communication Systems (NCCR-MICS), a center supported by the Swiss National Science Foundation under grant number 500567322 .

${ }^{2}$ One can show that at rates above the critical rate $E_{B}\left(R, Q_{Y \mid X}\right)$ exceeds the sphere packing bound and in particular that $\max _{x, x^{\prime}} D\left(Q_{Y \mid x} \| Q_{Y \mid x^{\prime}}\right) \geq C\left(Q_{Y \mid X}\right)$. and a stopping time $U(M)$ relative to the received symbols $Y_{1}, Y_{2}, \ldots$ The decoded message is $\psi_{U(M)}^{M}\left(y_{1}^{U(M)}\right)$. A coding scheme is a tuple $\mathcal{S}^{M}=\left(\Phi^{M}, \Psi^{M}, U(M)\right)$.

Definition 2 (Rate). For a given channel $Q_{Y \mid X}$, an integer $M \geq 2$ and a coding scheme $\mathcal{S}^{M}=\left(\Phi^{M}, \Psi^{M}, U(M)\right)$, the average rate is

$$
R\left(\mathcal{S}^{M}, Q_{Y \mid X}\right)=\frac{\ln M}{\mathbb{E} U(M)} \text { nats per symbol . }
$$

The limiting rate for a sequence of coding schemes $\theta=$ $\left\{\mathcal{S}^{M}\right\}_{M \geq 2}$ and a given channel $Q_{Y \mid X}$ is given by

$$
R\left(\theta, Q_{Y \mid X}\right)=\liminf _{M \rightarrow \infty} R\left(\mathcal{S}^{M}, Q_{Y \mid X}\right) .
$$

The average error probability, over uniformly chosen messages, given a coding scheme $\mathcal{S}^{M}$ and a channel $Q_{Y \mid X}$ is denoted by $\mathbb{P}\left(\mathcal{E} \mid Q_{Y \mid X}, \mathcal{S}^{M}\right)$.

Definition 3 (Error Exponent). Given a sequence of coding schemes $\theta=\left\{\mathcal{S}^{M}\right\}_{M \geq 2}=\left\{\Phi^{M}, \Psi^{M}, U(M)\right\}_{M \geq 2}$ the error exponent is

$$
E\left(\theta, Q_{Y \mid X}\right)=\liminf _{M \rightarrow \infty}-\frac{1}{\mathbb{E} U(M)} \ln \mathbb{P}\left(\mathcal{E} \mid Q_{Y \mid X}, \mathcal{S}^{M}\right) .
$$

Definition 4 (Optimal Sequences of Coding Schemes). Let $\mathcal{Q}$ be a family of DMC's. A set $\Theta$ of sequences of coding schemes is said to be optimal for $\mathcal{Q}$ if for any given constant $\nu$ with $0 \leq \nu<1$ there exists $\theta \in \Theta$ such that for any $Q_{Y \mid X} \in \mathcal{Q}$

$$
\begin{gathered}
E\left(\theta, Q_{Y \mid X}\right)=E_{B}\left(R\left(\theta, Q_{Y \mid X}\right), Q_{Y \mid X}\right) \\
\quad \text { and } R\left(\theta, Q_{Y \mid X}\right) \geq \nu C\left(Q_{Y \mid X}\right) .
\end{gathered}
$$

In other words a set $\Theta$ of sequence of coding schemes is optimal for a family of channels $\mathcal{Q}$ if for any a priori chosen fraction $0 \leq \nu<1$ there exists a sequence of coding schemes $\theta \in \Theta$ that simultaneously over $\mathcal{Q}$ satisfies the two following conditions. It achieves a rate at least equal to $\nu C\left(Q_{Y \mid X}\right)$ and has a corresponding error exponent equal to the maximum achievable error exponent that could be obtained if the channel statistics were revealed to both the encoder and the decoder.

Theorem. Let $L$ be any constant with $0 \leq L<1 / 2$. Let $\mathcal{Q}$ be the family of binary symmetric channels with crossover probability $\varepsilon$ with $0 \leq \varepsilon \leq L$. Then there exists a set $\Theta$ of optimal sequences of coding schemes for $\mathcal{Q}$.

The same result as above holds if the family $\mathcal{Q}$ represents now the set of $\mathrm{Z}$ channels with crossover probability $\varepsilon$ such that $0 \leq \varepsilon \leq L$ and with $0 \leq L<1$.

\section{ACKNOWLEDGMENTS}

The authors wish to thank E. Arikan and M.V. Burnashev for stimulating discussions and helpful comments.

\section{REFERENCES}

[1] M. V. Burnashev, "Data transmission over a discrete channel with feedback: Random transmission time" Problems of Information Transmission, vol. 12, number 4, p. 250-265, 1976. 